\title{
A utilização de um software infantil na terapia fonoaudiológica de Distúrbio do Processamento Auditivo Central
}

\author{
The use of a children software in the treatment of Central Auditory \\ Processing Disorder
}

\author{
Juliana Schwambach Martinss ${ }^{1}$, Maria Madalena Canina Pinheiro², Helena Ferro Blasi ${ }^{3}$
}

\begin{abstract}
RESUMO
O objetivo deste estudo foi verificar a efetividade do uso de recursos de informática na terapia fonoaudiológica do Distúrbio do Processamento Auditivo Central para a adequação das habilidades auditivas alteradas. Participaram desta pesquisa dois indivíduos, com diagnóstico do Distúrbio do Processamento Auditivo Central, sendo um do sexo masculino e outro do sexo feminino, ambos com nove anos. Os pacientes foram submetidos a oito sessões de terapia fonoaudiológica com a utilização do software e, posteriormente, realizou-se uma re-avaliação do processamento auditivo central para verificar o desenvolvimento das habilidades auditivas e a efetividade do treinamento auditivo. Verificou-se que, após o treinamento auditivo informal, houve adequação das habilidades auditivas de resolução temporal, figura-fundo para sons não verbais e verbais, ordenação temporal para sons verbais e não-verbais para ambos os pacientes. Conclui-se que o computador como instrumento terapêutico é um recurso estimulador e que possibilita o desenvolvimento de habilidades auditivas alteradas em pacientes com Distúrbio do Processamento Auditivo Central.
\end{abstract}

Descritores: Percepção auditiva; Estimulação acústica; Transtornos da percepção auditiva; Software

\section{INTRODUÇÃO}

$\mathrm{O}$ processamento auditivo central refere-se à funcionalidade que se fornece às informações auditivas recebidas ${ }^{(1)}$, sendo muito importante no desenvolvimento da linguagem e na construção de aprendizagens. Porém, para se dar funcionalidade a essas informações, precisa-se que as habilidades auditivas estejam integras e eficazes.

O Distúrbio do Processamento Auditivo Central (DPAC) acontece quando algo afeta, de forma adversa, o processamento da informação auditiva. Esse distúrbio faz com que ocorra uma dificuldade na interpretação dos padrões sonoros e pode conseqüentemente ocasionar prejuízos na compreensão das informações, alterações no comportamento e, em decorrência, o fracasso escolar.

Trabalho realizado na Clínica de Fonoaudiologia da Faculdade Estácio de Sá de Santa Catarina - FESSC - São José (SC), Brasil.

(1) Especializanda em Gerontologia pela Universidade Federal de Santa Catarina - UFSC - Florianópolis (SC), Brasil.

(2) Pós-graduanda em Distúrbios da Comunicação Humana pela Universidade Federal de São Paulo - UNIFESP - São Paulo (SP), Brasil; Professora do Curso de Fonoaudiologia da Faculdade Estácio de Sá de Santa Catarina FESSC - São José (SC), Brasil.

(3) Doutora, Coordenadora do Curso de Fonoaudiologia da Faculdade Estácio de Sá de Santa Catarina - FESSC - São José (SC), Brasil.

Endereço para correspondência: Juliana Schwambach Martins. R. Antenor Valentim da Silva, 1465/102, Jardim das Acácias, São José - SC, CEP 88111-

340. E-mail: juliana@fonobrasil.com.br

Recebido em: 8/1/2008; Aceito em: 17/4/2008
Na área da audição, especialmente nos DPAC, a Fonoaudiologia tem acompanhado as mudanças tecnológicas e desenvolvido software para reabilitar seus pacientes. Não se pode estagnar as técnicas terapêuticas e utilizar somente métodos tradicionais, pois as alterações encontradas em consultórios ao longo dos anos repetem-se; mas, os sujeitos que as possuem são totalmente diferentes, com histórias de vidas e necessidades terapêuticas distintas, além de um desenvolvimento e crescimento característicos do momento em que vivem.

A utilização do computador propicia inúmeros benefícios, pois possibilita o armazenamento e a organização da informação, além de ser um meio de comunicação interativo, oferece estímulo multi-sensorial (imagem, som, animação, virtualidade), diferentes oportunidades de aprendizagem; facilita as trocas de experiências e viabiliza diversas formas de expressão como: oral, escrita e visual ${ }^{(2)}$.

Acredita-se que a utilização do computador na clínica fonoaudiológica propicie uma abordagem terapêutica diferenciada e individualizada para esses pacientes. Salienta-se que esses pacientes são antes de tudo crianças que estão crescendo em um mundo repleto de tecnologias, entre elas o computador. O computador faz parte do cotidiano da maioria das crianças e sua utilização na terapia fonoaudiológica é estimulante para os pacientes, e essencial para os fonoaudiólogos. Além disso, o uso do computador proporciona o contato com um instrumento terapêutico que possibilita múltiplas estratégias que potencializam o desenvolvimento global e auditivo desses pacientes. 
Sendo assim, esse trabalho possuiu como objetivo geral verificar a efetividade do uso de recursos de informática na terapia fonoaudiológica do distúrbio do processamento auditivo central para a adequação das habilidades auditivas alteradas. Os objetivos específicos almejados foram: (a) apurar quais as habilidades auditivas e processos gnósicos se beneficiam com a utilização de recursos de informática; (b) comparar as avaliações do processamento auditivo central, realizadas antes e após o uso do computador e (c) conferir a viabilidade do computador na clínica fonoaudiológica.

\section{APRESENTAÇÃO DOS CASOS CLÍNICOS}

Essa pesquisa foi realizada na Clínica de Fonoaudiologia da Faculdade Estácio de Sá de Santa Catarina (FESSC), no período de fevereiro a outubro de 2007. Foi classificada quanto à natureza e aos procedimentos como experimental, segundo os seus objetivos como descritiva, pois descreve as características de uma população com DPAC. A amostragem caracteriza-se como não-probabilística intencional.

O estudo foi submetido e aprovado pelo Comitê de Ética e Pesquisa em Seres Humanos da mesma instituição sob o número de aprovação 030/2006.

A amostra foi formada por dois sujeitos com diagnóstico de Distúrbio do Processamento Auditivo Central (DPAC), sendo um do sexo masculino e outro do sexo feminino, ambos com idade de nove anos. Os sujeitos foram selecionados após análise de prontuários de pacientes com DPAC atendidos na Clínica de Fonoaudiologia da FESSC, tendo como critério a ausência de terapia fonoaudiológica pregressa ou atual. Foram selecionados dois pacientes com diferentes déficits gnósicos e habilidades auditivas, objetivando observar a efetividade do software na terapia.

Para participar do estudo os pacientes foram submetidos previamente à avaliação otorrinolaringológica, avaliação audiológica básica completa e avaliação do processamento auditivo central.

Os pacientes escolhidos foram descritos como paciente 1 (P1) e paciente 2 (P2). A P1 é do sexo feminino, possui nove anos de idade, e suas inabilidades auditivas são de figurafundo para sons verbais e não verbais; a resolução temporal e os déficits gnósicos são o não-verbal e o de decodificação. O P2 é do sexo masculino, possui nove anos e suas inabilidades auditivas são: ordenação temporal para sons verbais e não-verbais, resolução temporal e figura-fundo para sons verbais (integração binaural) e o grau de sua dificuldade foi classificado como severo. Os processos gnósicos alterados eram: decodificação, codificação (perda gradual da memória), organização e não-verbal.

Os pacientes foram submetidos à terapia fonoaudiológica individual, tendo a duração de oito sessões terapêuticas, com a utilização do software infantil "Pedro na casa malassombrada ${ }^{\circledR}$ ". Cada sessão terapêutica com o software infantil teve duração de 45 minutos, ocorrendo uma vez por semana. Na primeira sessão, realizou-se a assinatura do termo de consentimento livre e esclarecido pelos responsáveis pelos pacientes.

Este software tem o objetivo de contribuir para o desen- volvimento das habilidades de percepção auditiva de forma contextualizada. Este programa é composto por oito exercícios que envolvem diferentes padrões verbais e não verbais para o treinamento das seguintes habilidades auditivas: detecção sonora, reconhecimento e identificação do som, discriminação auditiva, análise e síntese auditiva, figura-fundo e ordenação temporal para sons verbais e não-verbais, contagem, seqüência de padrão de freqüência e duração, reconhecimento dos aspectos prosódicos lingüísticos, atenção e fechamento auditivo ${ }^{(3)}$.

Os resultados dos pacientes foram descritos por sessão terapêutica e nos diferentes cômodos propostos pelo software.

$\mathrm{Na}$ análise dos dados foi considerada como desempenho ruim porcentagem inferior a $60 \%$, regular a porcentagem de acertos igual ou maior a $60 \%$ até $70 \%$. Foi estimado como bom desempenho a porcentagem de acertos entre $71 \%$ a $100 \%$.

A primeira sessão terapêutica foi realizada no cômodo da sala no quadro do "grito". Nessa sessão a P1 obteve um bom desempenho para ordenar até três estímulos sonoros nãoverbais, e um desempenho regular para a ordenação a partir de três sons verbais.

Já, P2 apresentou um bom desempenho para ordenar até dois sons não-verbais, e para sequencializar até três sons verbais. Esse paciente não conseguiu sequencializar adequadamente esses estímulos, além de não se lembrar do primeiro som escutado, respondia com latência aumentada aos estímulos sonoros não-verbais. Provavelmente estes pacientes não realizaram adequadamente esta tarefa em virtude da dificuldade de memorizar mais elementos.

$\mathrm{Na}$ segunda sessão terapêutica foram realizadas as atividades ambientadas no banheiro e na cozinha da casa. Nessas tarefas tanto P1 como P2, obtiveram um bom desempenho para discriminar padrões de frequiência e de duração, porém apresentaram maior facilidade para os padrões de duração. Reforçase assim, que essa facilidade na realização da atividade ocorreu devido aos estímulos não necessitarem de ordenação.

Na terceira sessão terapêutica foram utilizadas as atividades da escada e em seguida na sala de música da residência. $\mathrm{Na}$ atividade da escada ambos os pacientes obtiveram um bom desempenho, sendo assim equivalentes. Na sala de música P1 apresentou um bom desempenho para ordenar a música com três seqüências, porém seu desempenho foi ruim para ordenar quatro sequiências. Já, P2 apresentou desempenho ruim e muita dificuldade para realizar esta tarefa tanto com três como com quatro sequiências, demonstrando assim dificuldades nos aspectos prosódicos.

Na quarta sessão terapêutica, visitou-se o quarto e o sótão da habitação na atividade do baú. Na atividade do quarto ambos os pacientes apresentaram um bom desempenho para discriminar os estímulos verbais.

$\mathrm{Na}$ atividade do sótão-baú, ambos obtiveram um bom desempenho tanto para reconhecer as vozes como para as expressões, porém, $\mathrm{P} 2$ obteve melhor aproveitamento do que P1 para reconhecer as expressões, visto que, seu percentual de acertos foi superior ao da mesma.

A quinta sessão terapêutica foi ambientada na sala de música no quadro do "homem". Verificou-se que P1 obteve um bom desempenho da ordenação de até três estímulos sonoros não-verbais, um desempenho regular na ordenação de quatro 
sons e um desempenho ruim para ordenar cinco sons.

O P2 apresentou um bom desempenho para ordenar até três sons não-verbais e um desempenho ruim para ordenar quatro e cinco sons. Vale ressaltar que P2 antes da terapia apresentava dificuldade para ordenar sons não-verbais. Este software novamente mostrou-se um importante auxílio para a melhora desta habilidade auditiva que requer integração inter-hemisférica.
Na sexta sessão terapêutica visitou-se a sala da residência, na qual está exposto o quadro da "Monalisa". P1 obteve um bom desempenho para ordenar todos os sons não-verbais, enquanto que P2 apresentou um bom desempenho para ordenar até três sons e um desempenho regular na ordenação de quatro e cinco sons não-verbais.

A sétima sessão terapêutica contextualizou-se no sótão da habitação, mais especificamente na atividade do esqueleto.

Tabela 1. Distribuição do desempenho percentual dos pacientes na primeira avaliação de processamento auditivo e na reavaliação após a terapia fonoaudiológica com o uso do software.

\begin{tabular}{|c|c|c|c|c|c|c|}
\hline Testes & $\begin{array}{c}1^{\circ} \text { Avaliação } \\
\text { P1 } \\
16 \text { e } 23 / 10 / 06\end{array}$ & $\begin{array}{c}1^{\circ} \text { Avaliação } \\
\text { P2 } \\
11 \text { e } 18 / 04 / 07\end{array}$ & $\begin{array}{c}2^{\circ} \text { Avaliação } \\
\text { P1 } \\
\text { 19/06/07 }\end{array}$ & $\begin{array}{c}2^{\circ} \text { Avaliação } \\
\text { P2 } \\
\text { 10/10/07 }\end{array}$ & $\begin{array}{c}\text { Desempenho } \\
\text { final } \\
\text { P1 }\end{array}$ & $\begin{array}{c}\text { Desempenho } \\
\text { final } \\
\text { P2 }\end{array}$ \\
\hline LS & Normal & Normal & & & Normal & Normal \\
\hline \multirow[t]{2}{*}{ MSV } & Normal & 3 síl.= Normal & & & & \\
\hline & & 4 síl.= 1/3 Aterado & & 4 síl.= 3/3 Normal & Normal & Normal \\
\hline MSNV & Normal & Normal & & & Normal & Normal \\
\hline \multirow[t]{2}{*}{ FRB } & $\mathrm{OD}=80 \%$ & $\mathrm{OD}=88 \%$ & & & & \\
\hline & $\mathrm{OE}=84 \%$ & $\mathrm{OE}=76 \%$ & & & Normal & Normal \\
\hline \multicolumn{7}{|l|}{ PSI } \\
\hline PSI-MCC (F/R=0) & Normal & Normal & & & Normal & Normal \\
\hline PSI-MCC(F/R=-40) & Normal & Normal & & & & \\
\hline PSI-MCI (F/R=0) & Normal & Normal & & & & \\
\hline $\mathrm{PSI}-\mathrm{MCl}(\mathrm{F} / \mathrm{R}=-10)$ & Normal & Normal & & & & \\
\hline RGDT & $\begin{array}{l}500 \mathrm{~Hz}=>40 \mathrm{~ms} \\
1000 \mathrm{~Hz}=40 \mathrm{~ms} \\
2000 \mathrm{~Hz}=>40 \mathrm{~ms} \\
4000 \mathrm{~Hz}=>40 \mathrm{~ms} \\
\text { Alterado= }=40 \mathrm{~ms}\end{array}$ & $\begin{array}{l}500 \mathrm{~Hz}=2 \mathrm{~ms} \\
1000 \mathrm{~Hz}=5 \mathrm{~ms} \\
2000 \mathrm{~Hz}=25 \mathrm{~ms} \\
4000 \mathrm{~Hz}=10 \mathrm{~ms} \\
\text { Alterado=10,5 ms }\end{array}$ & $\begin{array}{l}500 \mathrm{~Hz}=15 \mathrm{~ms} \\
1000 \mathrm{~Hz}=5 \mathrm{~ms} \\
2000 \mathrm{~Hz}=40 \mathrm{~ms} \\
4000 \mathrm{~Hz}=2 \mathrm{~ms} \\
\text { Alterado } 15,5 \mathrm{~ms}\end{array}$ & $\begin{array}{l}500 \mathrm{~Hz}=2 \mathrm{~ms} \\
1000 \mathrm{~Hz}=10 \mathrm{~ms} \\
2000 \mathrm{~Hz}=10 \mathrm{~ms} \\
4000 \mathrm{~Hz}=10 \mathrm{~ms} \\
\text { Média }=8 \mathrm{~ms}\end{array}$ & $\begin{array}{l}\text { Alterado, } \\
\text { porém com } \\
\text { melhora } \\
\text { expressiva. }\end{array}$ & Normal \\
\hline TDD & $\begin{array}{l}\mathrm{OD}=70,0 \% \text { alterado } \\
\mathrm{OE}=70,0 \%\end{array}$ & & $\begin{array}{l}\mathrm{OD}=96,25 \% \text { Normal } \\
\mathrm{OE}=95 \%\end{array}$ & & Normal & \\
\hline TDNV & $\begin{array}{l}\text { Atenção Seletiva } \\
\text { OD }=7 \text { acertos } \\
\text { OE }=17 \text { acertos } \\
\text { Alterado à OD } \\
\text { Atenção Sustentada } \\
\text { Não realizado }\end{array}$ & Normal & $\begin{array}{l}\text { Atenção Seletiva } \\
O D=14 \text { acertos } \\
O E=10 \text { acertos } \\
\text { Atenção Sustentada } \\
O D=11 \text { acertos } \\
O E=11 \text { acertos }\end{array}$ & & Normal & Normal \\
\hline
\end{tabular}

TPD

3 sons

4 sons
Normal

OD $=70 \%$ - Alterado

$\mathrm{OE}=70 \%$ - Alterado
OD $=100 \%-$

Normal

$\mathrm{OE}=100 \%$ -

Normal

Aspectos
quantitativos:
Normal
$95 \%$
$90 \%$ - Normal
$75 \%$ - Alterado -
Grau Moderado
$95 \%$

Aspectos

Aspectos

quantitativos: baixo/

alto

$93,3 \%$

$50 \%$ - Alterado

$72,5 \%$ - Alterado -

Grau Severo

$92,5 \%$
Normal

Alterado,
orém com
melhora
expressiva.

Legenda: LS: Localização sonora; MSV: Memória seqüencial verbal; MSNV: Memória seqüencial não-verbal; FRB: Fala com ruído branco; PSI: Teste pediátrico de inteligibilidade de palavras; RGDT: Randon gap detection test, TDD: Teste dicótico de dígitos; TDNV: Teste dicótico não-verbal; TPD: Teste padrão de duração; SSW: Teste de dissílabos alternados 
Nessa sessão P1 apresentou total aproveitamento, pois acertou todos os sons verbais propostos, assim como P2, e ambos obtiveram um bom desempenho nas habilidades de discriminação, reconhecimento e prosódia.

Na oitava sessão terapêutica, ambientada na sala de música da residência, realizou-se a atividade que segundo os resultados encontrados no decorrer da terapia fonoaudiológica, foi classificada como mais defasada no desempenho dos sujeitos. Verificou-se que P1 apresentou um bom desempenho para ordenar as músicas com três e quatro seqüências. P2 apresentou um bom desempenho para sequenciar três sons e rendimento ruim para sequenciar quatro sons.

Com essa atividade finalizou-se a terapia fonoaudiológica utilizando o software infantil Pedro na Casa Mal Assombra$\mathrm{da} \AA$, para ambos os pacientes.

Após um mês do término da terapia os pacientes foram reavaliados. Na sequiência apresenta-se a Tabela 1, com a exposição dos testes realizados com os pacientes na primeira bateria de testes do processamento auditivo central, isto é, antes da realização da terapia fonoaudiológica. São apresentados também na Tabela 1 os testes realizados após a estimulação auditiva. Cabe ressaltar que só foram reaplicados os testes alterados na primeira avaliação. Inicialmente, descrevem-se os resultados obtidos na reavaliação de P1 e posteriormente de $\mathrm{P} 2$.

Verificou-se que na primeira avaliação P1 possuía os seguintes testes alterados: Teste de Detecção de Gap Randomizado (RGDT), Teste Dicótico de Dígitos (TDD) e Teste Dicótico Não-Verbal (TDNV) na etapa de atenção livre. Esses testes verificam as seguintes habilidades auditivas: resolução temporal, figura-fundo para sons verbais e figura-fundo para sons não-verbais.

Após a terapia fonoaudiológica verificou-se que com P1 ocorreu uma total efetividade de treinamento para a habilidade de figura-fundo, tanto para sons verbais como para sons nãoverbais, visto que se adequou essa habilidade auditiva que se encontrava prejudicada anteriormente. Quanto à habilidade de resolução temporal verificou-se uma melhora significativa, porém essa ainda encontra-se alterada.

Verificou-se que na primeira avaliação P2 possuía os seguintes testes alterados: Memória Seqüencial Verbal (MSV), Teste Padrão de Duração (TPD), RGDT e Teste de Dissílabos Alternados (SSW). Com base nos resultados da aplicação destes testes constatou-se que P2 possuía as seguintes habilidades auditivas prejudicadas: ordenação temporal para seqüência simples, ordenação temporal para sons não-verbais, resolução temporal e figura-fundo para sons verbais.

Para o P2 o treinamento auditivo foi efetivo na adequação das habilidades de ordenação temporal para sons verbais e não-verbais e resolução temporal. Quanto às habilidades de figura-fundo para sons verbais e de integração binaural, houve uma melhora expressiva, porém ambas encontram-se ainda alteradas.

\section{DISCUSSÃO}

No presente estudo observou-se que os pacientes desenvolveram sua atenção auditiva e gradativamente conseguiram assimilar as informações retidas na sua memória. Os jogos contidos no computador auxiliaram os sujeitos a desenvolverem suas habilidades auditivas e a construírem novas possibilidades de aprendizagem.

Na primeira sessão terapêutica observou-se que à medida que aumentavam a quantidade de estímulos, acrescia-se a dificuldade dos indivíduos para realizarem a habilidade de ordenação temporal de sons verbais e não-verbais É importante ressaltar que a habilidade auditiva de ordenação temporal requer a aprendizagem de habilidades prévias como o reconhecimento, discriminação e memória auditiva. Um ambiente de estimulação auditiva adequado, bem como a experienciação, é fundamental para o desenvolvimento desta habilidade tão importante no processo de comunicação.

Na segunda sessão terapêutica, na tarefa vivenciada no banheiro, pode-se constatar que os pacientes encontraram maiores dificuldades para discriminar os padrões tonais de frequiência do que os de duração. Coincidindo com a literatura estudada $^{(4)}$ que encontrou em escolares de 10 anos um percentual de acerto de 53,3\% para a discriminação dos padrões de duração, enquanto que para os padrões de freqüiência esse percentual foi de $37,2 \%$, assim como os resultados encontrados nesse estudo.

$\mathrm{Na}$ atividade ambientada na cozinha, cabe ressaltar que para a P1 foi necessário explicar os conhecimentos sobre igualdade e diferença, para só então iniciar a atividade infere-se, então, que P1 apresentava um atraso no desenvolvimento de linguagem, visto que além da ausência desses conhecimentos possuía um vocabulário restrito. Acredita-se que alterações nas habilidades auditivas de atenção seletiva, detecção do som, sensação sonora, discriminação, localização sonora, reconhecimento, compreensão e memória auditiva podem refletir no comportamento e na linguagem dos sujeitos.

$\mathrm{Na}$ terceira sessão terapêutica observou-se que, conforme afirmam alguns estudos, para ocorrer o processamento dos sons é necessário o trabalho conjunto das redes neuronais complexas e das funções mentais superiores, visto que essas interpretam os sons verbais e não-verbais.

Na quarta sessão terapêutica, ambientada na sala de música, chama atenção o fato de que P1 conhecia as músicas infantis e as cantava durante as ordenações, como a música "Sambalelê". Já, P2 não reconheceu e não cantou nenhuma música. Acredita-se que esse conhecimento prévio demonstrado por P1 possa ter interferido nos resultados, pois a estimulação auditiva constante e progressiva propicia inúmeros benefícios para a compreensão dos sons, tanto verbais como não-verbais, além de ser extremamente importante para a plasticidade e para o aprendizado. A plasticidade cerebral é um fenômeno dinâmico que produz mudanças físicas estruturais, bioquímicas e comportamentais refletindo na modificação da função cerebral e alterando a efetividade das conexões existentes ${ }^{(5)}$.

Alguns autores afirmam que o tratamento para os DPAC faz-se necessárias modificações ambientais, estratégias compensatórias, e motivacionais; e atividades diretas na terapia que focalizem as habilidades auditivas alteradas e motivem a neuroplasticidade ${ }^{(6)}$. Na terapia fonoaudiológica pode ser utilizado apoio compensatório na fase inicial, porém como nesta pesquisa era necessário averiguar efetividade do software, 
foram retiradas as estratégias compensatórias dos pacientes.

A aprendizagem é um processo de aquisição de novos conhecimentos que posteriormente serão armazenados na memória $^{(7)}$. Fato este que provavelmente auxiliou a P1 a apresentar melhor resultado do que o P2 nas atividades que envolviam o armazenamento dos estímulos auditivos.

Na quinta e na sexta sessões terapêuticas verificou-se que a habilidade de ordenação temporal para sons verbais e nãoverbais exigiu dos pacientes uma maior concentração e atenção frente aos estímulos sonoros. Infere-se que provavelmente o fato da P1 não apresentar dificuldade na avaliação inicial de ordenação temporal auxiliou esta a apresentar melhor desempenho na sexta sessão terapêutica, pois além de realizar figura-fundo nesta tarefa também foi estimulada a habilidade de ordenação temporal. P2 apresenta, na avaliação inicial, inabilidade tanto de figura-fundo como de ordenação temporal, justificando seu pior desempenho nessa atividade. Entende-se que este recurso foi válido para treinar as habilidades auditivas defasadas dos pacientes de uma forma lúdica.

Na sétima sessão terapêutica P1 mostrou-se tranqüila e bastante concentrada apresentando assim uma mudança em seu comportamento, além de estar envolvida ludicamente com o jogo. Já, P2 manteve o comportamento calmo e tranqüilo em todas as estimulações auditivas.

A reabilitação informal pode auxiliar nessa aprendizagem e ser realizada com programas de computador, visto que é indicada para indivíduos com DPAC, que esses necessitam de diversos estímulos auditivos para exercitar as habilidades de ordenação temporal e de memória ${ }^{(8)}$. Tal afirmação vem corroborar com os resultados do presente estudo que mostra a eficácia deste programa para treinamento da ordenação temporal e memória.

Os recursos de informática auxiliam e motivam o desenvolvimento das habilidades de comunicação dos pacientes com alterações auditivas. $\mathrm{O}$ sucesso do treinamento auditivo dependerá da escolha criteriosa do software e da disposição do paciente ${ }^{(9-13)}$. Ressalta-se que a tecnologia pode auxiliar o terapeuta a desenvolver sua capacidade clínica e assim alcançar os objetivos do tratamento terapêutico.

Na oitava sessão a P1 novamente cantou todas as melodias das cantigas infantis juntamente com o estímulo não-verbal, como: "Marcha Soldado" e "Borboletinha". Essa paciente modificou sua postura corporal, e ficou sentada em uma postura adequada e não mais debruçada por sobre o computador. Já o P2 demonstrou muita dificuldade e insegurança para ordenar corretamente as músicas e não conhecia nenhuma canção. Porém ao se comparar os resultados da oitava sessão com a terceira em que foi trabalhada a mesma atividade sendo ela a sala de música, verificou-se uma melhora significativa da ordenação temporal para sons não-verbais em ambos os pacientes. Esta atividade mostrou-se válida para trabalhar tal habilidade auditiva e o déficit gnósico do tipo não-verbal, para o qual é necessário o processamento da informação não-verbal pelos dois hemisférios cerebrais.

Especificamente no Teste de Detecção de Gap Randomizado (RGDT) cada paciente melhorou a habilidade de resolução temporal de acordo com a freqüência testada. Em um estudo semelhante, cujo objetivo era verificar a habilidade de resolu- ção temporal em 15 escolares, sendo oito do sexo masculino e sete do sexo feminino, com a faixa etária de sete a 13 anos de idade, os valores médios obtidos nas freqüências de 500 $\mathrm{Hz}, 1 \mathrm{kHz}, 2 \mathrm{kHz}$ e $3 \mathrm{kHz}$ foram respectivamente 7,47 ms; $8,67 \mathrm{~ms} ; 8,07 \mathrm{~ms}$ e $10,93 \mathrm{~ms}^{(7)}$. Estes autores evidenciaram ainda que a habilidade de resolução temporal reflete-se diretamente na capacidade do sujeito de diferenciar os aspectos supra-segmentares da fala. Com isso, os autores concluíram que a habilidade de resolução temporal ocorreu de maneira diferenciada, de acordo com a freqüência testada, fato que se encontrou também no presente estudo.

A maturação do corpo caloso tem sido apontada como um fator que influencia na realização dos testes dicóticos ${ }^{(10)}$. Acredita-se que tal maturação tenha acontecido com P1, devido às respostas encontradas na re-avaliação desse tipo de teste com a mesma.

A efetividade do treinamento auditivo está associada à plasticidade do sistema nervoso central aliado à estimulação auditiva. Em um estudo a fim de verificar a manutenção das habilidades auditivas em 20 indivíduos, com a faixa etária de oito a 24 anos, os pesquisadores verificaram se após seis meses do treinamento auditivo as habilidades auditivas melhoradas mantiveram-se ou pioraram novamente. Da amostra estudada, 17 mantiveram a melhora obtida com o treinamento, porém dois não mantiveram essa melhora e um sujeito piorou o DPAC. Os pesquisadores ressaltam ainda que a melhora ocorrida nos pacientes estimulados se deve ao fato do treinamento auditivo ser um "método rápido, eficaz e não invasivo, que melhora a qualidade de vida, auxilia no desempenho escolar e na interação familiar e social"(11).

Sobre o Teste SSW, observou-se que os resultados encontrados com P2 coincidiram com os de uma pesquisa realizada em 95 crianças com a faixa etária de nove a dez anos de idade, com e sem dificuldades escolares e/ou das habilidades auditivas. Esse estudo objetivou caracterizar e comparar o desempenho dos sujeitos utilizando o teste SSW e concluiu que ocorreram mais erros quando havia condições competitivas do que em situações não competitivas; que esses erros ocorreram mais em indivíduos com nove anos; o pico de erros foi nas colunas E e F; e que a habilidade auditiva de figura-fundo para sons verbais melhorou conforme o crescimento e a maturação neurológica. Tal estudo confere com a presente pesquisa, visto que, em situações competitivas $\mathrm{P} 2$, também de nove anos de idade apresentou maiores erros, especialmente na coluna $\mathrm{F}^{(12)}$.

Em uma pesquisa realizada com o objetivo de investigar a efetividade do treinamento auditivo formal no DPAC, fez-se um estudo de caso com um indivíduo de nove anos, sendo do sexo masculino e que possuía como queixa distúrbio de aprendizagem. Nessa pesquisa, comprovou-se a efetividade do treinamento auditivo formal, por meio de testes objetivos, observando-se uma melhora na latência na onda do P300. A estimulação auditiva propiciou melhoras significativas nos resultados dos exames e refletiu-se no seu desempenho escolar e no relacionamento familiar do sujeito estudado ${ }^{(14)}$.

Por último, entregou-se para os seus responsáveis pelos pacientes os relatórios e pediu-se um depoimento dos mesmos sobre a importância da terapia fonoaudiológica e suas implicações na vida dos seus filhos. 
No depoimento concedido pela mãe da P1, a mesma proferiu as seguintes palavras, quando questionada sobre a evolução da sua filha e sobre a terapia Fonoaudiológica:

"Ela tinha muita dificuldade, não se concentrava, a gente chamava e ela estava longe (...) não ia tão bem nas provas. $E$ agora a gente percebe que ela consegue pegar um livro, ler e ficar quieta. A terapia ajudou bastante e eu gostei".

Após a reavaliação dos testes alterados na bateria de processamento auditivo central constatou-se o treinamento auditivo realiza mudanças comportamentais ${ }^{(6)}$.

A responsável pelo sujeito $\mathrm{P} 2$ em seu depoimento disse:

"Ele mudou muito se comparado ao que ele era, até as notas melhoraram. Ele tinha dificuldade em tudo para entender agora vejo uma diferença enorme. A terapia eu gostei, foi bastante produtiva e ajudou bastante".

Com base nesses depoimentos, infere-se que esse período de treinamento auditivo tenha estimulado e desenvolvido as habilidades auditivas, auxiliando-os assim a modificar seus comportamentos perante a escola e seus familiares. $\mathrm{O}$ treinamento auditivo na reabilitação os DPAC tem demonstrado, por meio de pesquisas e relatórios clínicos, a melhora das habilidades auditivas ${ }^{(15)}$.

Acredita-se que indivíduos que realizam treinamento auditivo apresentam alterações comportamentais, as quais podem decorrer devido à plasticidade cerebral.

\section{CONSIDERAÇÕES FINAIS}

Diante da análise dos resultados, pode-se concluir que a terapia fonoaudiológica para o DPAC utilizando o software "Pedro na casa mal assombrada®" foi efetiva para a adequação das habilidades auditivas alteradas.
Após terapia fonoaudiológica, houve adequação das habilidades auditivas de resolução temporal, figura-fundo para sons não verbais e verbais, ordenação temporal para sons verbais e não-verbais. Os processos gnósicos que apresentaram melhora nos sujeitos P1 e P2 foram o do tipo não-verbal, organização e codificação (perda gradual da memória). O déficit gnósico de decodificação apresentou melhora expressiva, porém ambos permaneceram com alteração nesse processo.

Verificou-se que os testes que apresentaram melhora no desempenho após a reavaliação de P1 foram: o Teste Dicótico de Dígitos (TDD) e o Teste Dicótico Não-Verbal (TDNV); e para P2 foram os testes: Teste de Detecção de Gap Randomizado (RGDT) e Teste Padrão de Duração (TPD).

Observaram-se mudanças expressivas nos resultados obtidos nas reavaliações do DPAC, se comparadas antes e após a terapia, além de perceber mudanças no comportamento social e auditivo de ambos os pacientes.

Reabilitar e intervir nos DPAC são papéis do fonoaudiólogo, da família e da escola, que juntos formam um alicerce para que o portador dessa alteração desenvolva suas potencialidades e supere as dificuldades auditivas, que refletem em toda sua vida.

Perante os dados obtidos nesse estudo, ressalta-se a importância de realizar uma intervenção fonoaudiológica diferenciada, eficaz e motivadora em pacientes com DPAC. Conclui-se que o computador utilizado como instrumento terapêutico, é um recurso estimulador que possibilita o desenvolvimento de habilidades auditivas prejudicadas em pacientes com DPAC.

O computador faz parte do contexto infantil e do terapêutico, porém inseri-lo na terapia para o DPAC foi um desafio que produziu conhecimento, modificou comportamentos, adequou e sanou dificuldades auditivas.

\begin{abstract}
The aim of this study was to verify the effectiveness of the use of computer science resources in the treatment of Central Auditory Processing Disorder, in order to adequate the altered auditory abilities. Two individuals with diagnosis of Central Auditory Processing Disorder, a boy and a girl, both with nine years old, participated on this study. The subjects were submitted to eight sessions of speech therapy using the software and, after this period, a reassessment of the central auditory processing abilities was carried out, in order to verify the development of the auditory abilities and the effectiveness of the auditory training. It was verified that, after this informal auditory training, the auditory abilities of temporal resolution, figure-ground for both verbal and nonverbal sounds, and temporal ordering for verbal and nonverbal sounds were adequate for both subjects. It was possible to conclude that the use of computers as therapeutical instruments is a stimulating resource that allows the development of altered auditory abilities in patients with Central Auditory Processing Disorder.
\end{abstract}

Keywords: Auditory perception; Acoustic stimulation; Auditory perceptual disorders; Software

\title{
REFERÊNCIAS
}

1. Katz K, Wilde L. Tratado de audiologia clínica. 4a ed. São Paulo: Manole, 1999. Capítulo 32, Desordens do processamento auditivo. p. 486-98.

2. Foz FBA. (Re)-evolução tecnológica e o aprendizado da leitura e da escrita. Fono Atual. 2002;5(22):5-7.
3. Santos RM, Toffoli MB, Cardoso APB, Drumond GP, da Rosa DA, coordenadores. Pedro na casa mal-assombrada: desenvolvendo habilidades auditivas. Manual do usuário. Ribeirão Preto: Book Toy Brinquedos e Livros; 2006. 
4. Gimenes VD, Pereira LD. Teste de padrão tonal de duração e freqüência sonora e habilidade grafofônicas. Pró-Fono. 2002;14(2):205-14.

5. Barreto MASC, Muniz LF, Teixeira CF. Desempenho da habilidade de resolução temporal em crianças de 7 a 13 anos. Rev Soc Bras de Fonoaudiol. 2004;9(4):220-8.

6. Bellis TJ. Auditory processing disordes: it's not just kids who have them. Hear J. 2003;56(5):17-8.

7. Kandeell ER, Schwartz JH, Jessell TM. Fundamentos da neurociência e do comportamento. Rio de Janeiro: Guanabara Koogan; 1997. p. 520- 30.

8. Musiek FE. Temporal (auditory) training for (C) APD. Hear J. 2005;58(2):46.

9. Bloom S. Technologic advances raise prospects for a resurgence in use of auditory training (cover story). Hear J. 2004;57(8):19-23.

10. Musiek FE, Gollegly KM. Maturational considerations in the neuroauditory evaluation of children. In: Bess $\mathrm{H}$, editor. Hearing impairment in children. Parkton (MD): York Press; 1988. p. 231-50.
11. Schochat E, Carvalho LZ, Megale RL. Treinamento auditivo: avaliação da manutenção das habilidades. Pró-Fono. 2002;14(1):93-8.

12. Câmara CC, Pereira LD, Borges ACLC. Teste de escuta dicótica de dissílabos - SSW - em crianças com e sem evidências de problemas escolares e/ou alteração das habilidades auditivas. Fono Atual. 2004;7(30):4-13.

13. Van Vliet D. In audiology, as in life, technology is necessary, but it's not enough. Hear J. 2005;58(9):88-9.

14. Kozlowski L, Wiemes GMR, Magni C, Silva ALG. A efetividade do treinamento auditivo na desordem do processamento auditivo central: estudo de caso. Rev Bras Otorrinolaringol. 2004;70(3):427-32.

15. Musiek FE. Auditory training and CPAD: a short history. Hear J. 2006;59(8):52. 